\title{
Platelet Gel in the treatment of generalized aggressive periodontitis: A case report
}

\section{Mriganka Barman Chowdhuri}

Assistant Professor, Army Dental Centre Research \& Referral Delhi, India

*Corresponding Author:

Email: mrig_chow@yahoo.com

\begin{abstract}
Aggressive periodontitis is a less prevalent, multifactorial disease which characteristically results rapid destruction of periodontium and early tooth loss. This is more frequently seen in systemically healthy younger age group of patients. Aggressive periodontitis can manifest in localized or generalized form. Generalized aggressive periodontitis is clinically characterized by loss of interproximal attachment of at least three permanent teeth apart from first molars and incisors. Conventional surgical procedure with autologous platelet Gel and systemic antibiotics were used successfully to treat and manage this case of generalized aggressive periodontitis.
\end{abstract}

Keywords: Aggressive Periodontitis (AP), Generalized Aggressive Periodontitis (GAP). Autologous Platelet Gel (PG). Platelet Rich Plasma (PRP).

\section{Introduction}

Aggressive Periodontitis (AP) being a multifactorial disease usually results rapid destruction of Periodontium. This disease is reported to affect less than $1 \%$ of population and commonly seen in systemically healthy individuals under 30 years of age. Older patients with AP also reported in the literature. ${ }^{1}$ the amount of local etiological factors also characteristically do not corroborate with the severity of periodontal tissue destruction. Various microbiologic, genetic, immunologic and environmental/behavioural risk factors were identified but our understandings about the pathogenicity is still limited. The terminology of AP has been updated many times over the years from diffuse atrophy of the alveolar bone in 1923 to be finally named as aggressive periodontitis in 1999. ${ }^{2}$ AP are now described in two forms as Localized Aggressive Periodontitis (LAP) that mainly affects the permanent first molars and incisors; and Generalized Aggressive Periodontitis (GAP) that involves interproximal attachment loss of three or more permanent teeth along with first molars and incisors. ${ }^{3} \mathrm{~A}$ case of GAP managed successfully by conventional surgical procedure using Autologous Platelet Gel (PG) and systemic antibiotics is reported here.

\section{Case Report}

A 26-year-old male presented with complaints of bleeding from gum, halitosis, increased spacing of upper and lower anterior teeth, dull pain and occasional purulent discharge from gingiva for last six months. Individual was non-smoker unmarried and free of systemic ailments which was further substantiated by general examination, detail blood and urine examination. Family history was positive for Type-II diabetes and primary hypertension. Intra-oral examination revealed full complement of teeth with mesio-angular impaction of 38 and 48. Grade-I mobility of maxillary and mandibular incisors were observed clinically with few carious lesions in upper and lower molars without pulpal involvement. Gingiva was soft and oedematous with ulcerated rolled up bulbous margin and fiery red in colour (Fig. 1). There were generalized bleeding on probing and loss of stippling. Periodontal probing revealed average pocket depth (PD) more than $7 \mathrm{~mm}$ in all four quadrants. Maximum recorded PD in max incisors were $9 \mathrm{~mm}$. PD in the mesial aspects in 36 and 46 were $8 \mathrm{~mm}$ and $9 \mathrm{~mm}$ respectively. Maximum recorded $\mathrm{PD}$ in maxillary molars and incisors were $7 \mathrm{~mm}$ and $8 \mathrm{~mm}$ respectively. Plaque and calculus were present but visibly did not corroborate with the amount of gingival inflammation present. Orthopantomogram revealed generalized bone loss with both vertical and horizontal component and characteristically more prominent in maxillary and mandibular 1st molars and incisors areas (Fig. 2). Microbiological investigation of plaque samples revealed gram negative black pigmented indole positive porphyromonas species (Fig. 3). On the basis of clinical, radiological and microbiological investigations, diagnosis of GAP was made.

\section{Management}

Etiotropic phase-I therapy was carried out with scaling, root planning in multiple successive appointments. Oral hygiene instructions with sulcus brushing (modified Bass technique) advised. Twice daily $(10 \mathrm{ml}) 0.2 \%$ Chlorhexidine mouth wash $(\mathrm{CHX})$ advised along with systemic antibiotic Doxycycline 100 $\mathrm{mg}$ once daily for 14 days. Definite reduction in probing depths and gingival inflammation observed on revaluation after 4 weeks. Full-mouth flap surgery was performed addressing one quadrant each time after raising full thickness flap with sulcular incision. Autologous platelet Gel (PG) prepared from Platelet Rich Plasma (PRP) applied to the bone defects and planed root surface. ${ }^{4}$ Periodontal pack was applied after apposition of flap with sutures. Patient was prescribed 
requisite analgesics and $500 \mathrm{mg}$ of amoxicillin thrice daily along with metronidazole $500 \mathrm{mg}$ twice daily for 7 days. Postoperative healing was satisfactory and uneventful. Sutures were removed on 8th day. Regular follow up of the patient carried out. After 14 weeks, there were no signs of bleeding on probing and the probing depths were also within normal limits (Fig. 4). During the further follow-up up to 11 months there were no signs of recurrence and the patient was able to maintain the oral hygiene adequately.

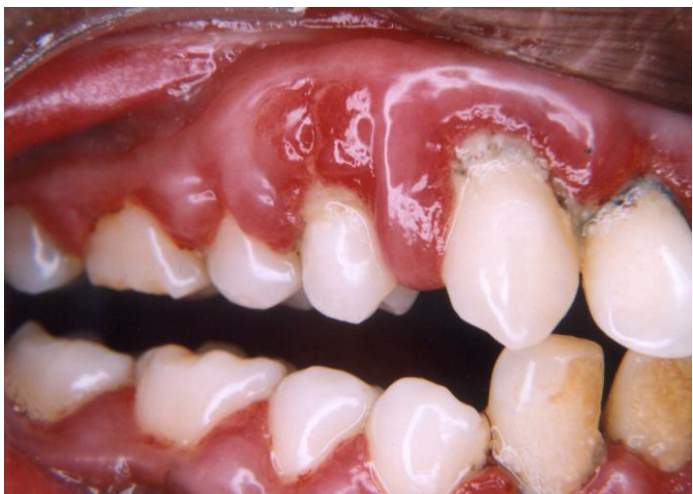

Fig. 1: Preoperative clinical presentation with generalized inflamed, spongy and bleeding gingival tissues with loss of physiologic architectures (Use colours)

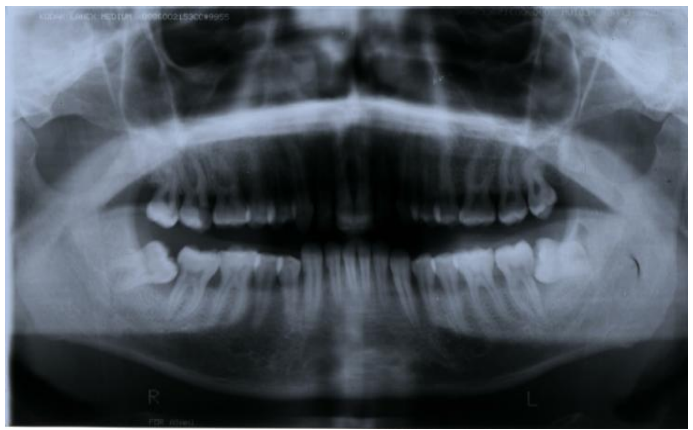

Fig. 2: Orthopantomogram (OPG) revealing characteristic generalized bone loss

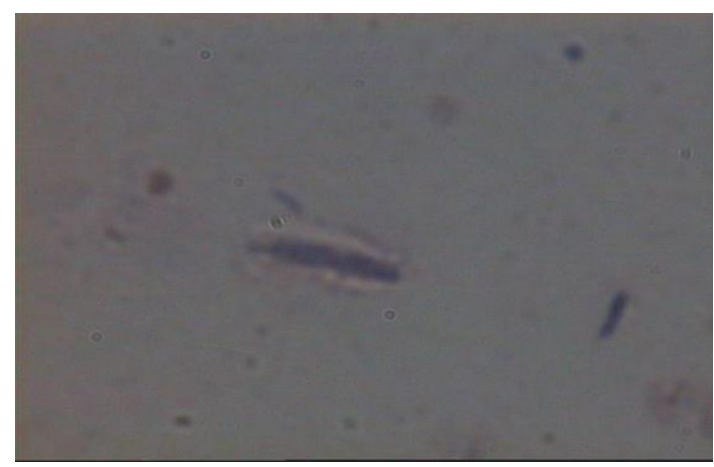

Fig. 3: Black pigmented Porphyromonas species under electron microscope (Use colours).

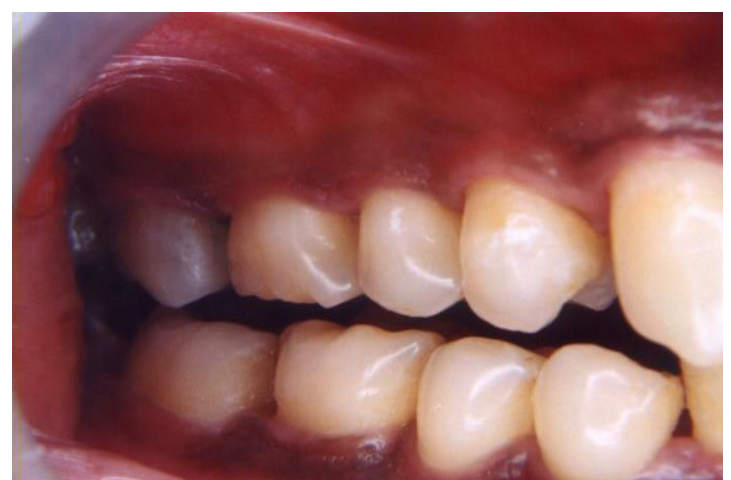

Fig. 4: Postoperative clinical presentation of healthy gingival tissues (Use colours)

\section{Discussion}

Management of GAP patients is traditionally pursued with nonsurgical therapy, periodontal surgical therapy and lifelong supportive and maintenance therapy. The treatment outcome depends on early diagnosis that helps in the prevention of extensive attachment and bone loss. The disease in the early stage are usually managed by nonsurgical therapy along with systemic antibiotics. Open flap debridement carried out either with Modified Widman's flap or Kirkland's sulcular incision flap design along with or without resective or regenerative procedures. These surgical procedure eliminate periodontal pockets and establish healthy self-maintainable periodontal environment. ${ }^{5,6}$ Regenerative techniques consist of Guided Tissue Regeneration (GTR) with bone graft alone or in combination with biologic mediators like plateletderived growth factor (PDGF) and extracellular matrix proteins like enamel matrix protein (EMD). ${ }^{7}$ The meticulous supportive periodontal therapies are essential for further maintenance and clinical improvements.

PG being autologous is safe and cost effective. It is easy to use and consists of a fibrin matrix of platelets, leukocyte, cytokines and stem cells. PG being rich source of PDGF, TGF, Vascular Endothelial Growth Factors (VEGF) and Epithelial Growth Factors (EGF) promotes bone regeneration and soft tissue healing and definitely reduce bony defect in GAP. ${ }^{8}$ Systemic antibiotics are used along with definite periodontal therapy in the treatment of AP because of specific association of pathogens such as Aggregatibacter actinomycetemcomitans and Porphyromonas gingivalis to the disease and usually provide greater clinical improvement. The preferred and first-line combination antibiotic therapy at present for treatment of GAP is $250 \mathrm{mg}$ of amoxicillin three times daily with metronidazole $250 \mathrm{mg}$ two times daily for 8 days. ${ }^{9}$ Doxycycline, Azithromycin Metronidazole and Clindamycin when used alone found effective as an adjunct to nonsurgical therapy in AP patients. $^{10}$ Management of Aggressive periodontitis is indeed more challenging due to specific bacterial involvement 
subjective and varied host responses and genetic predispositions. Further advance researches are required to understand the aetiology and pathogenesis of these disease which in turn will help us formulating better treatment plan.

\section{References}

1. American Academy of Periodontology. Parameter on aggressive periodontitis. J Periodontol. 2000;71(5):86769.

2. Guzeldemir E, Toygar HU. From alveolar diffuse atrophy to aggressive periodontitis: a brief history. J Hist Dent. 2006;54(3):96-99.

3. Lang N, Bartold PM, Cullinan M, et al. Consensus report: aggressive periodontitis. Ann Peridontol. 1999;4(1):53.

4. Tozum TF, Demiralp B. Platelet rich plasma: a promising innovation in dentistry. J Can Dent Association. 2003:69(10):664.

5. Ramfjord SP, Nissle RR. The modified widman flap. $J$ Periodontol. 1974;45(8):601-7.

6. Kirkland O. The supportive periodontal therapy. J Am Dent Association 1931;18:1460-70.
7. Polson AM, editor. Periodontal Regeneration: Current Status and Directions. $1^{\text {st }}$ ed. Quintessence. 1994.

8. Plachokova AS, Nikolidakis D, Mulder J, Jansen JA, Creugers NHJ. Effect of platelet-rich plasma on bone regeneration in dentistry: a systematic review. Clin Oral Implants Res. 2008;19(6):539-45.

9. Griffiths GS, Ayob R, Guerrero A et al. Amoxicillin and metronidazole as an adjunctive treatment in generalized aggressive periodontitis at initial therapy or retreatment: a randomized controlled clinical trial. J Clin Periodontol. 2011;38(1):43-9.

10. Xajigeorgiou C, Sakellari D, Slini T, Baka A, Konstantinidis A. Clinical and microbiological effects of different antimicrobials on generalized aggressive periodontitis. J Clin Periodontol. 2006;33(4):254-64.

How to cite this article: Chowdhuri M B. Platelet Gel in the treatment of generalized aggressive periodontitis: A case report. Int $\mathrm{J}$ Periodontol Implantol. 2018;3(4):137-139. 